\title{
Sintomas depressivos e déficit cognitivo na população de 60 anos e mais em um município de médio porte do interior paulista*
}

Depression symptoms and cognitive-deficit in a population aged 60 years and older in a medium-sized city in São Paulo state, Brazil

Síntomas depresivos y de déficit cognitivo en la población de 60 años y más en una ciudad de tamaño medio en el estado de São Paulo, en Brasil

José Evandro Marques Gomes ${ }^{1 * *}$, Tania Ruiz², José Eduardo Corrente ${ }^{3}$

Palavras-chave: Saúde do Idoso Depressão Transtornos Mentais Manifestações Neurocomportamentais Avaliação Geriátrica

\section{Resumo}

Introdução: a população mundial está envelhecendo e o Brasil segue essa tendência, o que demanda uma reorganização da sociedade para o cuidado desses idosos. Observa-se, nesta tendência, um aumento do número de casos de depressão e demência, além da vinculação destas com outras doenças crônico-degenerativas. Objetivo: estimar a prevalência dos sintomas depressivos e déficits cognitivos em uma população de 60 anos e mais, moradora de um município de médio porte do interior do estado de São Paulo, e sua associação com outras doenças crônico-degenerativas mais prevalentes. Métodos: estudo transversal, com 364 idosos, utilizando: instrumentos sociodemográficos e de morbidade; o Mini Exame do Estado Mental (MEEM), a Escala de Yesavage, a Escala de Atividades de Vida Diária e a Escala de Atividades Instrumentais de Vida Diária (AIVD). Foram realizadas: análises estatísticas de frequências dos escores dos instrumentos; apresentação da sumarização das variáveis e as possíveis associações entre depressão/demência, aplicando-se 0 teste do $\chi^{2}$ seguido do ajuste de um modelo de regressão logística para dados ordinais. Resultados: a suspeita de depressão foi encontrada em $44 \%$ (160) e o déficit cognitivo foi identificado em 38,7\% (141) dos idosos. Aproximadamente $75 \%$ dos idosos, com suspeita de depressão ou déficit cognitivo, eram portadores de pelo menos mais uma patologia crônica. Foi possível estabelecer associações estatisticamente significativas entre: suspeita de depressão e AIVD ( $p<0,0001 ; 0 R=7,59$; IC=3,361-7,139) e déficit cognitivo e AIVD ( $\mathrm{p}=0,0007 ; \mathrm{R}=3,967 ; \mathrm{IC}=1,788-8,799)$. Não foram encontradas associações entre idade, situação conjugal, escolaridade, inserção no mercado de trabalho, aposentadoria ou renda. Conclusão: idosos de ambos os sexos estão vulneráveis a doenças como depressão e demência. Por outro lado, sintomas depressivos e déficit cognitivo foram associados ao escore dos idosos comprometidos, segundo as AIVD.

\footnotetext{
Médico de Família e Comunidade de São Carlos (SP), Brasil. E-mail: koringa@estadao.com.br

2 Universidade Estadual Paulista (UNESP). E-mail: truiz@fmb.unesp.br

${ }^{3}$ Universidade Estadual Paulista (UNESP). E-mail: jecorren@ibb.unesp.br

*Parte da Dissertação de Mestrado apresentada à Faculdade de Medicina de Botucatu para a obtenção do Título de Mestre em Saúde Pública.

**Autor correspondente.

Fonte de financiamento: este projeto de pesquisa teve financiamento da Fapesp, com número de processo 2005/02510-0.

Conflito de interesses: declararam não haver.

Recebido em: 26/11/2010. Aprovado em: 12/05/2011
} 


\begin{abstract}
Keywords: Abstract
Health of the Elderly

Depression

Mental Disorders

Neurobehavioral Manifestations

Geriatric Assessment

Introduction: the world population is ageing, and Brazil follows this tendency, which requires the reorganization of society for care provision to older people. In such tendency, an increasing number of cases of depression and dementia is observed in addition to their association with other chronic-degenerative diseases. Objective: to estimate the prevalence of depression and cognitive-deficit symptoms in a population aged 60 years and older, residing in a middle-sized city in São Paulo state and to associate the population with other more prevalent chronic degenerative diseases. Methods: cross-sectional study on 364 older people using the following instruments: socio-demographic and morbidity, Mini Mental State Examination, Yesavage Scale, the Activities of Daily Living Scale, and the Instrumental Activities of Daily Living (IADL) Scale. The following were performed: statistical analyses of the instruments' score frequencies; presentation and summarization of the variables; and the possible associations between depression/dementia by applying the $\chi^{2}$ test followed by fitting of a logistic regression model for ordinal data. Results: the suspected depression was found in 44\% (160), and cognitive deficit was observed in $38.7 \%$ (141) aged. About $75 \%$ of the individuals with suspicion of depression or cognitive deficit had at least another chronic pathology. It was possible to establish statistically significant associations between suspected depression and IADL ( $p<0.0001 ; O R=7.59 ; C l=3.361-7.139)$ and cognitive deficit and IADL $(p=0.0007 ; O R=3.967 ; C l=1.788-8.799)$. No associations were found between age, marital status, schooling, placement in the work market, retirement or income. Conclusion: male and female older individuals are vulnerable to diseases, such as depression and dementia. On the other hand, depression symptoms and cognitive deficit were associated with the score of compromised older individuals, according to IADL.
\end{abstract}

Palabras clave:

Salud del Anciano

Depresión

Trastornos Mentales

Manifestaciones Neurocomportamentales

Evaluación Geriátrica

\section{Resumen}

Introducción: la población mundial está envejeciendo y Brasil sigue esta tendencia, que requiere una reorganización de la sociedad para el cuidado de los ancianos. Acepta-se que este número creciente de casos de la depresión y la demencia están asociados con otras enfermedades crónicas. Objetivo: estimar la prevalencia de los síntomas depresivos y deterioros cognitivos en una población de 60 años y más de edad, residente en una ciudad de tamaño medio en el Estado de São Paulo y su asociación con las enfermedades crónicas más frecuentes. Métodos: estudio transversal con 364 pacientes, utilizando los siguientes instrumentos: variables socio-demográficas y de morbilidad, el Mini Examen del Estado Mental, Yesavage Escala y Escala de Actividades de la Vida y Actividades Instrumentales de la Vida Diaria. Se realizaron: análisis estadísticos de frecuencias de las puntuaciones de los instrumentos; la presentación de los resúmenes de las variables y se estudiaran las posibles asociaciones entre la depresión y la demencia mediante la aplicación de la prueba de $\chi^{2}$ seguido por el ajuste de un modelo de regresión logística para datos ordinales. Resultados: la depresión fue encontrada en el 44\% (160) y el deterioro cognitivo fue identificado en 38,7\% (141) de las personas mayores. En aproximadamente el $75 \%$ de los pacientes ancianos con depresión, también se sospechó de deterioro cognitivo en los pacientes que tuvieron al menos una enfermedad más crónica. Fue posible establecer asociaciones estadísticamente significativas entre: sospecha de depresión y AIVD ( $p<0,0001,0 R=7.59$, IC=3,361$7,139$ ) y déficit cognitivo y AIVD ( $\mathrm{p}=0,0007 ; \mathrm{R}=3,967 ; \mathrm{IC}=1,788-8,799)$. No se encontró asociación entre sospecha de depresión y déficit cognitivo con la edad, el estado civil, la educación, la entrada en el mercado de trabajo, los ingresos o la jubilación. Conclusión: los adultos mayores de ambos los sexos son vulnerables a enfermedades como la depresión y la demencia. Por otra parte, los síntomas depresivos y el déficit cognitivo fueron asociados a la puntuación de los ancianos comprometidos, segundo el AIVD.

\section{Introdução}

No Brasil, ao contrário dos países desenvolvidos, o processo de transição demográfica se caracteriza pela rapidez do aumento absoluto e relativo das populações adultas e idosas, resultante do declínio da fecundidade e não da mortalidade ${ }^{1,2}$. Dados de 2006, do Instituto Brasileiro de Geografia e Estatística $(\mathrm{IBGE})^{3}$, mostram que a expectativa de vida média do brasileiro cresceu, atingindo 72,3 anos, sendo 76,1 anos para mulheres e 68,5 anos para homens. Entretanto, o envelhecer traz consigo perdas na função normal do organismo, aumentando, assim, o risco de doenças ${ }^{4}$.

Alguns autores ${ }^{5}$ mencionam que, de acordo com as pesquisas norte-americanas, $40 \%$ dos maiores de 65 anos apresentam alguma incapacidade, tendo a doença crônica como a principal causa. Já Negri et al. ${ }^{2}$ relatam que $80 \%$ dos idosos apresentam pelo menos uma enfermidade crônica. Dentre elas, é possível destacar as doenças cardiovasculares, o câncer, a obesidade, o diabetes, a depressão, a demência, entre outras $^{5}$. O envelhecimento pode também aumentar ou estar associado ao aparecimento de sintomas depressivos e déficits cognitivos, sendo que, frequentemente, estes sintomas não recebem diagnósticos ou tratamento ${ }^{6}$.

Neste contexto, destaca-se a depressão como um problema de saúde mental altamente prevalente no idoso, com consequências sociais e econômicas, sendo, portanto, de grande relevância para a saúde pública ${ }^{7}$. Estima-se em $5 \%$ a prevalência da depressão na população geral brasileira, sendo ainda maiores, 5 a $10 \%$, nos pacientes ambulatoriais, e entre 9 a $16 \%$ dos pacientes internados ${ }^{8}$. Porém, para Ferrari e Dalacorte 9 , a prevalência de idosos com sintomas depressivos é ainda maior, sendo $15 \%$ na comunidade, $20 \%$ na atenção primária e 20 a $25 \%$ nos hospitais.

Segundo o critério do Manual Diagnóstico e Estatístico de Transtornos Mentais, em sua quarta versão (DSM-IV$\mathrm{TR})^{10}$, o episódio depressivo maior caracteriza-se pela presença de, no mínimo, cinco dos seguintes sintomas, por um período de duas semanas. São eles: 
- humor deprimido na maior parte do dia, na maioria dos dias, observado por terceiros ou por relato subjetivo;

- diminuição do interesse ou prazer pela maioria das atividades a maior parte do dia, na maioria dos dias;

- perda ou ganho de mais de $5 \%$ do peso corporal em um mês;

- insônia ou hipersonia, na maioria dos dias;

- agitação ou retardo psicomotor quase todos os dias;

- diminuição da energia quase todos os dias;

- sentimentos de desvalia ou culpa excessiva, na maioria dos dias;

- dificuldades para pensar, concentrar-se ou tomar decisões quase todos os dias;

- pensamentos recorrentes sobre morte, planos ou tentativas de suicídio.

Para o diagnóstico, é importante também lembrar que a sintomatologia não deve satisfazer os critérios para episódio misto, não ser decorrente do uso de substâncias químicas ou condições médicas e não ter relação com luto. Além disso, os sintomas devem trazer sofrimento e prejuízos na qualidade de vida do indivíduo.

No caso da demência, ainda existem poucos estudos a respeito ${ }^{11}$. Segundo Machado et al. ${ }^{12}$, a demência também é um problema de saúde mental que vem aumentando paralelamente ao envelhecimento da população, com um incremento na incidência anual de $0,6 \%$ entre 65 a 69 anos, e de $8,4 \%$ naqueles com mais de 85 anos. Sua prevalência aumenta exponencialmente com a idade, passando de $5 \%$, naqueles com mais de 60 anos, para $20 \%$ após os 80 anos.

Em um estudo sobre a prevalência de demência, que foi realizado em uma cidade do interior do estado de São Paulo, este indicador variou de $1,3 \%$, na faixa etária entre 60 a 69 anos, a $36,9 \%$, para a faixa etária de 85 anos ou mais ${ }^{13}$. Outro estudo, realizado na clínica de memória da Santa Casa de São Paulo, entre fevereiro de 1997 e maio de 1998, verificou que 69,3\% dos pacientes portadores de quadros demências apresentaram sintomas característicos de depressão ${ }^{14}$. Em estudo de caso-controle, realizado na cidade do Rio de Janeiro, com 404 indivíduos com 60 anos e mais, constatou-se que idosos com quadros de demências apresentaram maior risco de queda e de serem hospitalizados do que aqueles sem demências ${ }^{15}$.

Para a DSM-IV-TR, a demência é caracterizada por múltiplos déficits cognitivos, os quais incluem o comprometimento da memória sem afetar a consciência. As funções cognitivas que estão comprometidas na demência incluem: inteligência geral; aprendizagem e memória; afasia (dificuldade na evocação dos nomes de pessoas e objetos); capacidade de resolução de problemas; orientação; sensopercepção; atenção; concentração; juízo crítico; agnosias (fracasso em reconhecer ou identificar objetos, apesar de função sensorial intacta); apraxias (prejuízo na capacidade de executar atividades motoras, apesar das capacidades motoras, as funções sensoriais e a compreensão estarem intactas para a tarefa exigida); funções executivas e habilidades sociais. A personalidade está comprometida. Um diagnóstico de demência para o DSM-IV-TR requer que os sintomas resultem em um comprometimento significativo do desempenho social e laborativo, e que eles representem um declínio significativo no nível anterior de desempenho. Portanto, enfatiza-se que as deficiências adquiridas das capacidades cognitivas prejudiquem o desempenho das atividades da vida diária, em comparação com o nível anterior de desempenho para que o diagnóstico seja justificado.

Teng, Humes e Demetrio ${ }^{16}$ analisaram a relação entre a depressão e algumas condições clínicas e estabeleceram algumas associações: maior mortalidade associada a sintomas depressivos e doenças crônicas; menor adesão a tratamentos clínicos em deprimidos; a presença de depressão triplica os gastos médicos; a presença de comorbidades clínicas com transtornos depressivos aumenta mais os dias de incapacitação do que a soma dos efeitos individuais das doenças clínicas.

Scalco et al ${ }^{17}$ também descrevem a associação entre depressão e hipertensão arterial, enquanto associação entre diabetes e depressão foi descrita por Musselmann et al. ${ }^{18}$, e a associação entre depressão e eventos cardíacos foi descrita por Bush et al. ${ }^{19}$; Teng, Humes e Demétrio ${ }^{16}$.

Para demência, encontram-se na literatura associações, mostrando uma relação de causa e efeito entre doenças crônicas e déficit cognitivo. Almeida-Pititto, Almada Filho e Cendoroglo $^{20}$ e Okereke et al ${ }^{21}$ descrevem a associação entre diabetes e déficit cognitivo, enquanto Saxby et al. ${ }^{22}$, Melo e Martin ${ }^{23}$ descrevem a associação entre hipertensão e déficit cognitivo.

Assim, o objetivo deste trabalho foi estimar a prevalência dos sintomas depressivos e déficit cognitivo; estimar a prevalência das doenças crônico-degenerativas e estudar as associações entre as patologias mais prevalentes na população de pacientes com 60 anos e mais em um município de médio porte, do interior do estado de São Paulo.

\section{Métodos}

As informações utilizadas neste trabalho provêm de bancos de dados produzidos por estudo epidemiológico, de corte transversal, realizado no município de Botucatu, em São Paulo, entre 2003 e 2007, sendo parte integrante do estudo intitulado "Validade e Coerência de Instrumentos Utilizados em Avaliações Clínicas de Idosos”. Tal estudo foi apresentado ao Comitê de Ética da Faculdade de Medicina da Universidade Estadual Paulista (UNESP), campus de Botucatu, sob protocolo 1777/2005, tendo sido aprovado em 4 de abril de 2005 . 


\section{Sujeitos do estudo}

Indivíduos moradores do município de Botucatu com 60 anos ou mais.

\section{Tamanho da amostra}

O tamanho amostral foi determinado considerando confiabilidade de $95 \%$, erro amostral de $5 \%$ e prevalência não-conhecida. A amostra estimada foi de um mínimo de 372 indivíduos com 60 anos e mais a serem entrevistados.

\section{Seleção da amostra}

Os participantes foram selecionados por amostragem aleatória e proporcional entre os domicílios. A identificação dos domicílios foi realizada por meio de um cadastro de 9.000 famílias alocadas aleatoriamente, sorteadas na proporção 4/1 e, em seguida, foram selecionados, aleatoriamente neste cadastro, moradores com 60 anos ou mais.

\section{Períodos da pesquisa}

Realizou-se, em 2000, um estudo-piloto com 15 idosos não-pertencentes à amostragem, escolhidos aleatoriamente na população, com o objetivo de testar e adaptar os instrumentos a serem aplicados.

Em 2003, ocorreu a primeira etapa da pesquisa, na qual, após reformulações nos questionários, os domicílios alocados foram visitados e o próprio idoso ou seu cuidador foi entrevistado. Ao não estar o idoso no domicílio, houve o retorno do pesquisador num outro momento, em até três vezes. Após a terceira vez, a ausência do respondente no domić́lio foi admitida como perda amostral. Foram perdidos sete idosos e, deste modo, obteve-se uma amostra final entrevistada de 365 idosos.

No retorno ao trabalho de campo, entre maio e junho de 2006, durante as re-entrevistas e avaliando os dados de sobrevivência dos idosos, constatou-se que, entre esses 365 idosos, ocorreram 80 perdas por morte ou mudança de endereço.

Para a segunda etapa da pesquisa, durante agosto de 2006, realizou-se mais uma vez um estudo piloto com dez idosos, os quais foram entrevistados em seus domicílios. A finalidade da entrevista foi identificar a necessidade de ajustes nos novos instrumentos aplicados e como treinamento para os entrevistadores, de modo a minimizar a ocorrência de erros de preenchimento.
No período de abril a agosto de 2007, foram entrevistados 80 idosos, que foram selecionados por meio da metodologia de sorteio do banco de dados de 2003 para repor as perdas da amostra atual, constituídas por mortes e recusas.

Após a realização das entrevistas dos "80 novos idosos", verificou-se que um dos entrevistados tinha menos de 60 anos, reduzindo a amostra a 364 idosos.

\section{Instrumentos utilizados}

Foram utilizados, neste estudo, quatro instrumentos para avaliação funcional (Escala de Atividades Básicas da Vida Diária) ${ }^{24}$ e a The Physical Self-Maintenance Scale ${ }^{25}$, que foi utilizada como medida de Atividade Instrumental da Vida Diária; emocional (Escala de Depressão Geriátrica Abreviada $^{26}$ ) e cognitiva (Mini Exame do Estado Mental ${ }^{27}$ ), os quais foram acompanhados de um questionário de identificação e um outro sociodemográfico, além de um questionário de morbidade referida, já aplicado aos idosos, no estudo de 2003.

\section{Consolidação e análise dos dados}

Os dados foram digitados por um profissional treinado contratado. Em seguida, foram analisados eletronicamente por meio do software Statistical Package for the Social Sciences (SPSS ${ }^{\circledR}$ ) for Windows, versão 16.0. Foram realizadas análises de frequências dos escores dos instrumentos, apresentação da sumarização das variáveis e as possíveis associações entre depressão/demência pelo teste do $\chi^{2}$. Os dados foram submetidos também à regressão logística ordinal, considerando-se como desfechos sintomas depressivos e déficit cognitivo, como exposição à hipertensão arterial sistêmica (HAS), diabetes mellitus (DM), cardiopatia e associações entre elas, e como covariáveis de controle: sexo, faixa etária, situação conjugal, escolaridade, inserção no mercado de trabalho, aposentadoria, renda e outra fonte de renda.

Em todos os testes, considerou-se o nível de significância de $5 \%$ ou o valor de p correspondente.

\section{Resultados}

Dos 364 idosos da amostra, 59,1\%, (215) eram do sexo feminino e 40,9\% (149) do sexo masculino. Em relação à idade, $48,1 \%$ dos participantes (175) tinham entre 60 e 69 anos; 35,7\% (130) entre 70 e 79 anos; e 16,2\% (59), 80 anos ou mais. A idade dos idosos da amostra variou entre 60 
e 106 anos, tendo como média 71,31 $( \pm 7,8)$. Foram identificados 25,8\% (94) de idosos analfabetos; 45,6\% (166), com nível primário; 19,8\% (72), com nível secundário e $8,8 \%$ (32), com nível superior completo ou incompleto. Em relação ao estado civil, $57,4 \%$ (209) dos idosos eram casados ou viviam em união estável; 36,3\% (132) eram viúvos, separados, desquitados ou divorciados e 6,3\% (23) eram solteiros. Portanto, conclui-se que 42,6\% (155) dos idosos não tinham um companheiro. 80,5\% (293) dos idosos trabalhavam e 66,2\% (241) estavam aposentados. Em relação à renda, 32,7\% (119) dos idosos referiram receber mais do que três salários mínimos e 45,9\% (167) referiram receber até três salários mínimos, havendo ocorrido 21,4\% (78) de respostas sem informação nesse quesito.

O instrumento utilizado para investigação da depressão, Escala de Yesavage, mostrou escores que variaram entre 0 e 14, com média de 5,14 $( \pm 3,0)$. Considerando os escores iguais ou maiores a seis como positivos, encontraram-se $44 \%$ (160) dos idosos com suspeita de depressão, sendo $40,7 \%$ (148) com suspeita de depressão leve à moderada e 3,3\% (12), com suspeita de depressão grave. No instrumento utilizado para avaliação cognitiva, o minimental, os escores variaram entre 4 e 30, com média de 23,38 $( \pm 4,4)$. Considerando os escores de 19 para iletrados (sem escolaridade) e 23 para letrados (com pelo menos um ano escolar), apurou-se que $38,7 \%$ (141) tinham déficit cognitivo.

A distribuição das doenças crônicas em pessoas com déficit cognitivo e de sintomas depressivos é apresentada na Figura 1.

Para a medida da autonomia, por meio da Escala de Atividades de Vida Diária (AVD), considerando o escore até 5 como dependentes, foram encontrados $17 \%$ (62) dos idosos dependentes. Já, para a medida da autonomia por meio da Escala de Atividades Instrumentais de Vida Diária (AIVD), aceitando-se escore inferior a 17 , como indicativo de dependência, foram encontrados 14\% (51) dos idosos dependentes.

Figura 1. Percentual de sintomas depressivos e déficit cognitivo, segundo demais doenças crônico-degenerativas, na população de 60 anos ou mais. Botucatu, 2007.

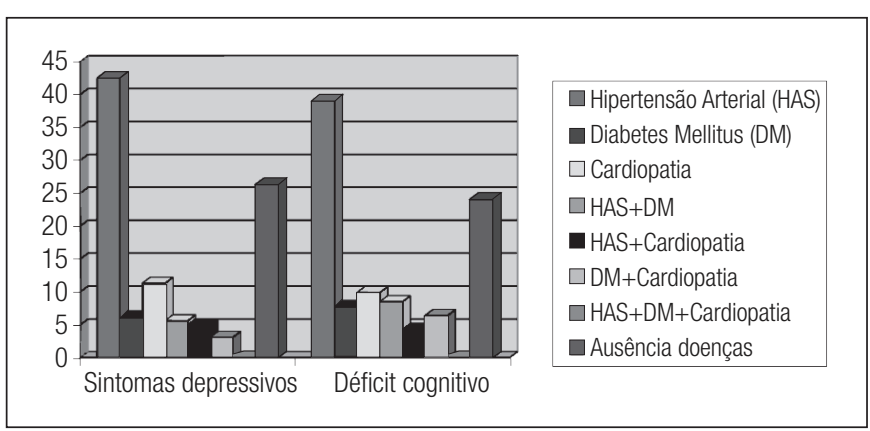

Tabela 1. Distribuição da população de 60 anos e mais, suspeitos de depressão, segundo características sociais, econômicas, patologias crônicas e dependência. Botucatu, 2007.

\begin{tabular}{|c|c|c|c|c|c|}
\hline $\begin{array}{l}\text { Características sociais } \\
\text { e econômicas }\end{array}$ & Total n (\%) & $\begin{array}{c}\text { Suspeita } \\
\text { Depressão (\%) }\end{array}$ & $p\left(x^{2}\right)$ & $\mathrm{OR}$ & IC (95\%) \\
\hline \multicolumn{6}{|l|}{ Sexo } \\
\hline Masculino & $146(40,8)$ & 43,2 & 0,626 & 1,111 & $(0,727 ; 1,699)$ \\
\hline Feminino & $212(59,2)$ & 45,8 & & 1,000 & \\
\hline \multicolumn{6}{|l|}{ Faixa etária } \\
\hline 70 anos e mais & $186(51,9)$ & 44,6 & 0,978 & 0,994 & $(0,655 ; 1,509)$ \\
\hline 60-69 anos & $172(48,1)$ & 44,8 & & 1,000 & \\
\hline \multicolumn{6}{|l|}{ Situação conjugal } \\
\hline Sem companheiro & $23(6,4)$ & 52,2 & 0,456 & 1,378 & $(0,591 ; 3,212)$ \\
\hline Com companheiro & $335(93,6)$ & 44,2 & & 1,000 & \\
\hline \multicolumn{6}{|l|}{ Escolaridade } \\
\hline Até 4 anos & $255(71,2)$ & 38,8 & 0,157 & 1,400 & $(0,878 ; 2,232)$ \\
\hline$>4$ anos & $103(28,8)$ & 47,1 & & 1,000 & \\
\hline \multicolumn{6}{|l|}{$\begin{array}{l}\text { Inserção no mercado } \\
\text { trabalho }\end{array}$} \\
\hline Não & $69(19,3)$ & 47,8 & 0,560 & 1,169 & $(0,691 ; 1,979)$ \\
\hline Sim & $289(80,7)$ & 43,9 & & 1,000 & \\
\hline \multicolumn{6}{|l|}{ Aposentadoria } \\
\hline Sim & $237(66,2)$ & 44,3 & 0,836 & 1,048 & $(0,675 ; 1,627)$ \\
\hline Não & $121(33,8)$ & 45,5 & & 1,000 & \\
\hline \multicolumn{6}{|l|}{ Renda* } \\
\hline Até $3 \mathrm{SM}$ & $167(46,6)$ & 29,9 & 0,419 & 0,813 & $(0,492 ; 1,344)$ \\
\hline$>3 \mathrm{SM}$ & $119(33,2)$ & 34,5 & & 1,000 & \\
\hline \multicolumn{6}{|l|}{ Outra fonte renda } \\
\hline Não & $311(86,9)$ & 45,0 & 0,752 & 1,105 & $(0,595 ; 2,054)$ \\
\hline Sim & $47(13,1)$ & 42,6 & & 1,000 & \\
\hline \multicolumn{6}{|l|}{ Hipertensão } \\
\hline Sim & $146(40,8)$ & 46,6 & 0,552 & 1,137 & $(0,744 ; 1,737)$ \\
\hline Não & $212(59,2)$ & 43,4 & & 1,000 & \\
\hline \multicolumn{6}{|l|}{ Diabetes } \\
\hline Sim & $22(6,2)$ & 45,5 & 0,941 & 1,033 & $(0,435 ; 2,457)$ \\
\hline Não & $336(93,8)$ & 44,6 & & 1,000 & \\
\hline \multicolumn{6}{|l|}{ Cardiopatia } \\
\hline Sim & $38(10,6)$ & 47,4 & 0,726 & 1,128 & $(0,575 ; 2,213)$ \\
\hline Não & $320(89,4)$ & 44,4 & & 1,000 & \\
\hline \multicolumn{6}{|c|}{ Hipertensão + diabetes } \\
\hline $\operatorname{Sim}$ & $22(6,2)$ & 40,9 & 0,713 & 0,848 & $(0,353 ; 2,038)$ \\
\hline Não & $336(93,8)$ & 44,9 & & 1,000 & \\
\hline \multicolumn{6}{|l|}{$\begin{array}{l}\text { Hipertensão + } \\
\text { cardiopatia }\end{array}$} \\
\hline Sim & $19(5,3)$ & 36,8 & 0,479 & 0,709 & $(0,273 ; 1,845)$ \\
\hline Não & $339(94,7)$ & 45,1 & & 1,000 & \\
\hline \multicolumn{6}{|l|}{ Diabetes + cardiopatia } \\
\hline Sim & $16(4,5)$ & 31,2 & 0,268 & 0,548 & $(0,187 ; 1,612)$ \\
\hline Não & $342(95,5)$ & 45,3 & & 1,000 & \\
\hline \multicolumn{6}{|c|}{$\begin{array}{l}\text { Hipertensão + diabetes } \\
\text { + cardiopatia }\end{array}$} \\
\hline $\operatorname{Sim}$ & $1(0,3)$ & 0,0 & 0,368 & 0,995 & $(0,985 ; 1,005)$ \\
\hline Não & $357(99,7)$ & 44,8 & & 1,000 & \\
\hline \multicolumn{6}{|l|}{ AVD } \\
\hline Dependente & $57(15,9)$ & 47,4 & 0,658 & 1,137 & $(0,645 ; 2,005)$ \\
\hline Independente & $301(84,1)$ & 44,2 & & 1,000 & \\
\hline \multicolumn{6}{|l|}{ AIVD } \\
\hline Dependente & $46(12,9)$ & 76,1 & 0,000 & 4,760 & $(2,330 ; 9,724)$ \\
\hline Independente & $312(87,1)$ & 40,1 & & 1,000 & \\
\hline \multicolumn{6}{|l|}{ Déficit cognitivo* } \\
\hline Sim & $159(44,4)$ & 38,4 & 0,768 & 0,938 & $(0,611 ; 1,438)$ \\
\hline Não & $198(55,3)$ & 39,9 & & 1,000 & \\
\hline
\end{tabular}

*alguns idosos não responderam todos os itens. Total de idosos analisados: 358 
Tabela 2. Distribuição da população de 60 anos e mais, com déficit cognitivo, segundo características sociais, econômicas, patologias crônicas e dependência. Botucatu, 2007.

\begin{tabular}{|c|c|c|c|c|c|}
\hline $\begin{array}{l}\text { Características } \\
\text { sociais e econômicos }\end{array}$ & Total n (\%) & $\begin{array}{c}\text { Déficit } \\
\text { Cognitivo (\%) }\end{array}$ & $\begin{array}{l}\text { Valor } \\
\text { de } p\end{array}$ & $\mathrm{OR}$ & IC (95\%) \\
\hline \multicolumn{6}{|l|}{ Sexo } \\
\hline Masculino & $145(40,5)$ & 38,6 & 0,807 & 1,055 & $(0,685 ; 1,626)$ \\
\hline Feminino & $213(59,5)$ & 39,9 & & 1,000 & \\
\hline \multicolumn{6}{|l|}{ Faixa etária } \\
\hline 70 anos e mais & $187(51,9)$ & 39,6 & 0,940 & 1,017 & $(0,665 ; 1,554)$ \\
\hline 60-69 anos & $171(47,8)$ & 39,2 & & 1,000 & \\
\hline \multicolumn{6}{|l|}{ Situação conjugal } \\
\hline Sem companheiro & $23(6,4)$ & 43,5 & 0,678 & 1,198 & $(0,510 ; 2,811)$ \\
\hline Com companheiro & $335(93,6)$ & 39,1 & & 1,000 & \\
\hline \multicolumn{6}{|l|}{ Escolaridade } \\
\hline Até 4 anos & $255(71,2)$ & 42,4 & 0,071 & 1,558 & $(0,962 ; 2,525)$ \\
\hline$>4$ anos & $103(28,8)$ & 32,0 & & 1,000 & \\
\hline \multicolumn{6}{|l|}{$\begin{array}{l}\text { Inserção no mercado } \\
\text { trabalho }\end{array}$} \\
\hline Não & $69(19,3)$ & 37,7 & 0,747 & 0,915 & $(0,533 ; 1,571)$ \\
\hline Sim & $289(80,7)$ & 39,8 & & 1,000 & \\
\hline \multicolumn{6}{|l|}{ Aposentadoria } \\
\hline Sim & $237(66,2)$ & 38,0 & 0,445 & 1,190 & $(0,762 ; 1,859)$ \\
\hline Não & $121(33,8)$ & 42,1 & & 1,000 & \\
\hline \multicolumn{6}{|l|}{ Renda* } \\
\hline Até 3 SM & $167(46,6)$ & 46,7 & 0,614 & 1,129 & $(0,704 ; 1,812)$ \\
\hline$>3 \mathrm{SM}$ & $119(33,2)$ & 43,7 & & 1,000 & \\
\hline \multicolumn{6}{|l|}{ Outra fonte de renda } \\
\hline Não & $311(86,9)$ & 40,2 & 0,421 & 1,302 & $(0,683-2,481)$ \\
\hline Sim & $47(13,1)$ & 34,0 & & 1,000 & \\
\hline \multicolumn{6}{|l|}{ Hipertensão } \\
\hline Sim & $146(40,8)$ & 37,7 & 0,582 & 0,886 & $(0,574 ; 1,365)$ \\
\hline Não & $212(59,2)$ & 40,6 & & 1,000 & \\
\hline \multicolumn{6}{|l|}{ Diabetes } \\
\hline Sim & $22(6,2)$ & 50,0 & 0,293 & 1,585 & $(0,668 ; 3,760)$ \\
\hline Não & $336(93,8)$ & 38,7 & & 1,000 & \\
\hline \multicolumn{6}{|l|}{ Cardiopatia } \\
\hline Sim & $38(10,6)$ & 36,8 & 0,734 & 0,886 & $(0,442 ; 1,778)$ \\
\hline Não & $320(89,4)$ & 39,7 & & 1,000 & \\
\hline \multicolumn{6}{|l|}{$\begin{array}{l}\text { Hipertensão + } \\
\text { diabetes }\end{array}$} \\
\hline Sim & $22(5,3)$ & 54,5 & 0,133 & 1,9256 & $(0,808 ; 4,584)$ \\
\hline Não & $336(94,7)$ & 38,4 & & 1,000 & \\
\hline \multicolumn{6}{|l|}{$\begin{array}{l}\text { Hipertensão + } \\
\text { cardiopatia }\end{array}$} \\
\hline Sim & $19(5,3)$ & 31,5 & 0,402 & 0,697 & $(0,258 ; 1,879)$ \\
\hline Não & $339(94,7)$ & 39,8 & & & \\
\hline \multicolumn{6}{|l|}{ Diabetes + cardiopatia } \\
\hline Sim & $16(4,5)$ & 56,2 & 0,158 & 2,045 & $(0,744 ; 5,624)$ \\
\hline Não & $342(95,5)$ & 38,6 & & 1,000 & \\
\hline \multicolumn{6}{|c|}{$\begin{array}{l}\text { Hipertensão + } \\
\text { diabetes + cardiopatia }\end{array}$} \\
\hline Sim & $1(0,3)$ & 0,0 & 0,420 & 0,605 & $(0,556 ; 0,657)$ \\
\hline Não & $357(99,7)$ & 39,5 & & 1,000 & \\
\hline \multicolumn{6}{|l|}{ AVD } \\
\hline Dependente & $56(15,5)$ & 51,8 & 0,039 & 1,822 & $(1,027 ; 3,234)$ \\
\hline Independente & $302(84,5)$ & 37,1 & & 1,000 & \\
\hline \multicolumn{6}{|l|}{ AIVD } \\
\hline Dependente & $45(12,6)$ & 71,1 & 0,000 & 4,607 & $(2,322 ; 9,142)$ \\
\hline Independente & $313(87,4)$ & 34,8 & & 1,000 & \\
\hline
\end{tabular}

*alguns dados não-preenchidos; total de idosos analisados: 358.
A Tabela 1 apresenta a distribuição dos idosos suspeitos de depressão e variáveis sociodemográficas, patologias e dependência.

A Tabela 2 apresenta a distribuição dos idosos suspeitos de déficit cognitivo e variáveis sociodemográficas, patologias e dependência.

Como é possível observar, nas Tabelas 1 e 2, existem três associações estatisticamente significativas. $\mathrm{Na}$ primeira, associando perda de autonomia e suspeita de depressão, $76,1 \%$ dos idosos dependentes eram suspeitos de depressão; a segunda associação encontrada foi entre déficit cognitivo e AVD, na qual 51,8\% dos idosos dependentes apresentavam déficit cognitivo. Finalmente, a última associação encontrada foi entre déficit cognitivo e AIVD, entre os idosos dependentes, $71,1 \%$ apresentavam déficit cognitivo. Observou-se também que a associação entre déficit cognitivo e hipertensão + diabetes, alcançou-se $\mathrm{p}=0,133$.

Por meio das análises de regressão logística, foi possível encontrar as associações que estão presentes nas Tabelas 3 e 4 .

Após o ajuste do modelo de regressão logística ordinal, verificou-se que as associações entre suspeita de depressão e AIVD e déficit cognitivo com AIVD se mantiveram estatisticamente significativas. A primeira foi aumentando sua força em 1,6 vezes, enquanto a segunda diminuiu sua força em 0,14 vezes. Entretanto, a associação de déficit cognitivo com AVD deixou de ser estatisticamente significativa e a associação entre déficit cognitivo e idosos portadores de hipertensão e diabetes, simultaneamente, quase se tornou estatisticamente significativa $(\mathrm{p}=0,0587)$.

\section{Discussão}

$\mathrm{Na}$ presente pesquisa, a suspeita de depressão foi averiguada em 44\% (160) dos idosos, sendo que 3,3\% (12) apresentaram suspeita de depressão grave e 40,7\% (148), de depressão leve à

Tabela 3. Ajuste do modelo de regressão logística ordinal para variáveis que se associaram à suspeita de depressão na população de 60 anos e mais. Botucatu, 2007.

\begin{tabular}{lccc}
\hline Associações & Valor de $\mathrm{p}\left(\mathrm{x}^{2}\right)$ & OR & IC \\
\hline Suspeita Depressão versus AIVD & $<0,0001$ & 7,59 & $(3,361-17,139)$ \\
\hline
\end{tabular}

Tabela 4. Ajuste do modelo de regressão logística ordinal para variáveis que se associaram ao déficit cognitivo na população de 60 anos e mais. Botucatu, 2007.

\begin{tabular}{lccc}
\hline Associações & Valor de $\mathrm{p}\left(\mathrm{x}^{2}\right)$ & $\mathrm{OR}$ & $\mathrm{IC}$ \\
\hline Déficit cognitivo versus AVD & 0.8429 & 1,069 & $(0,552-2,075)$ \\
\hline Déficit cognitivo versus AIVD & 0,0007 & 3,967 & $(1,788-8,799)$ \\
\hline Déficit cognitivo versus HAS + DM & 0,0587 & 3,211 & $(0,958-10,767)$ \\
\hline
\end{tabular}


moderada. Ferrari e Dalacorte ${ }^{12}$, ao estudarem idosos internados no Serviço de Geriatria do Hospital São Lucas da Pontifícia Universidade Católica do Rio Grande do Sul (PUCRS), e Oliveira, Gomes e Oliveira $^{28}$, ao estudarem idosos que frequentam centro de convivência, obtiveram uma prevalência muito próxima à deste estudo, 46 e 31\%, respectivamente. No entanto, outras pesquisas apontam prevalências mais baixas, como no estudo de Borges et al. ${ }^{29}$, que estudaram idosos cadastrados nos Centro de Saúde de Florianópolis, no qual foi utilizada a escala de Yesavage, e a mesma prevalência foi de $17,4 \%$, ficando muito próxima à prevalência referida na pesquisa de Paradela $e t$ al..$^{30}$. Estes autores estudaram idosos que frequentam unidade ambulatorial de saúde da rede pública da cidade do Rio de Janeiro, com prevalência de $16,9 \%$, em que também utilizou-se a mesma escala.

O déficit cognitivo foi identificado em $38,7 \%$ (141) dos idosos entrevistados nesta pesquisa, utilizando o Mini Exame do Estado Mental (MEEM). Na literatura estudada, é possível observar que diversos autores utilizam o MEEM, alguns deles utilizando esta escala como critério diagnóstico para demência, enquanto outros somente para avaliar déficits de cognição. Entre déficit cognitivo e demência, notam-se poucas variações de prevalência, a maior, $52,4 \%$, foi averiguada na pesquisa de Engelhardt et al..$^{31}$, quando estudaram idosos em asilo de alta qualidade do município do Rio de Janeiro, e a menor, 9,1\%, foi relatada na pesquisa de Borges et al. ${ }^{30}$, ao estudarem idosos cadastrados nos Centro de Saúde de Florianópolis.

Assim como a idade, a escolaridade também vem sendo descrita como característica importante para o aparecimento de depressão e demência. Para Lourenço e Veras ${ }^{32}$, a quantidade dos anos de estudo tem sido identificada como fator de proteção neural e como fator de confusão para o diagnóstico, uma vez que os instrumentos utilizados para avaliação cognitiva são altamente influenciados pela escolaridade. Neste estudo, foi obtido um resultado favorável a este argumento: para aqueles com mais de quatro anos de estudo, 32\% (33) dos pesquisados obtiveram resultado favorável ao déficit cognitivo, para os classificados em até quatro anos escolares, esse resultado foi de 42,4\% (108).

Para Borges et al. ${ }^{29}$, estudos recentes vêm mostrando relação entre escolaridade e depressão. Almeida Filho et al. ${ }^{33}$ constataram que baixa escolaridade tem maior influência no aparecimento de depressão em mulheres. Com base neste trabalho, é possível afirmar que as considerações feitas são válidas também no presente estudo, uma vez que, entre as pessoas pesquisadas com até quatro anos escolares, 47,1\% (120) eram suspeitos de depressão e, entre os pesquisados com mais de quatro anos escolares, $38,8 \%$ (40) foram considerados suspeitos de depressão.

Almeida Filho $e t$ al.$^{33}$ associam o fato de não estar casado (solteiro, viúvo ou divorciado) a menor prevalência de depressão no homem, já para as mulheres, ser solteira indicava menor prevalência de depressão. No entanto, para Paradela et al. ${ }^{30}$, a presença de um companheiro diminuiu a prevalência dos sintomas depressivos. Fato que o último autor relaciona com a solidão dos que vivem sozinhos, o que poderia aumentar as queixas de sintomas depressivos. Foram obtidos resultados muito próximos para a suspeita de depressão entre casados e não-casados: dos casados, $44,7 \%$ foram considerados suspeitos, dos sozinhos (viúvos, separados, divorciados e desquitados), o resultado apurado foi de $43,4 \%$, pouco abaixo dos solteiros com $52,2 \%$.

Verificou-se, no presente trabalho, que $76,1 \%$ dos idosos considerados dependentes na AIVD $(\mathrm{p}=0,00)$ apresentavam suspeita de depressão e, para a última escala, $71,1 \%$ dos dependentes apresentavam déficit cognitivo $(\mathrm{p}=0,00)$. Enquanto que para a Escala de Vida Diária, 51,8\% dos dependentes apresentavam déficit cognitivo. Na literatura, foi identificada como justificativa dos resultados mostrados, a qualidade de vida dos idosos. A independência física e financeira, as atividades de lazer e cultura e diversas outras dimensões da capacidade funcional, independentemente da presença ou não de enfermidades, podem proteger os idosos destas doenças ${ }^{34}$.

O resultado encontrado de AVD maior que AIVD é justificado, segundo Colombini Neto ${ }^{35}$, à pouca consistência interna da AVD devido à primeira questão apresentar a palavra "OU", a qual, por se tratar de uma questão composta por uma negativa, gera confusão nas respostas e, consequentemente, redução na coerência.

No estudo de Ferrari e Dalacorte ${ }^{9}$, observou-se que a média do escore do MEEM foi maior em pacientes cujo resultado da escala de AVD foi pleno do que naqueles com resultado não-pleno. Porém, não houve diferença significativa para os considerados deprimidos.

A presente pesquisa apurou também na associação entre HA e DM com déficit cognitivo $\mathrm{p}<0,06$. Pode ser que se o tamanho da amostra fosse aumentado, ele se tornasse estatisticamente significativo, já que existem fortes evidências de que as alterações vasculares, alterações do metabolismo de oxigênio, lesões endoteliais, efeitos tóxicos de subprodutos da insulina, entre outros explicariam este fenômeno.

Por fim, acredita-se que possa existir um viés pequeno, para mais, nas prevalências encontradas pelo fato de a amostra ter sido um retorno ao campo três anos depois do primeiro inquérito, apesar da substituição dos óbitos ter sido feita com idosos que tinham a mesma idade daqueles que morreram.

\section{Conclusão}

Com base nos resultados obtidos, pode-se concluir que idosos de ambos os sexos estão vulneráveis a doenças como 
depressão e demência, e que fatores como idade, situação conjugal, escolaridade, inserção no mercado de trabalho, aposentadoria e renda não foram encontrados neste estudo como associados ao desenvolvimento destas patologias.

Foi encontrada prevalência de suspeita de depressão e déficit cognitivo como descrito na literatura e aproximadamente $75 \%$ dos idosos com suspeita de depressão ou déficit cognitivo eram portadores de, pelo menos, uma patologia crônica. Por outro lado, verificou-se também que o nível de dependência dos idosos apareceu como fortemente associado à depressão e demência, sugerindo que o uso das escalas de AVD e AIVD pode ser uma potente ferramenta para a estratificação de risco para tais doenças.

Pode-se inferir, desta forma, que os profissionais de saúde deveriam aplicar uma Avaliação Geriátrica Ampla (Yesavage, MEEM, AVD ou AID) aos idosos sob sua responsabilidade, com o objetivo de melhorar a assistência à saúde; portanto, devendo receber treinamento e serem incentivados para tal.

É aconselhável também que políticas sociais para a infância e idade adulta preocupem-se com ações no sentido de que a população alcance a terceira idade com autonomia, levando-se em conta a alta magnitude das patologias crônicas e outras doenças causadoras de dependência, tais como depressão ou demência.

\section{Referências}

1. Carvalho J, Garcia RA. O envelhecimento da população brasileira: um enfoque demográfico. Cad Saúde Pública. 2003; 19(3): 725-33.

2. Negri LAS, Ruy GF, Collodeti JB, Pinto LF, Soranz DR. Aplicação de um instrumento para detecção precoce e previsibilidade de agravos na população idosa. Ciênc Saúde Coletiva. 2004; 9(4): 1033-46.

3. Instituto Brasileiro de Geografia e Estatística - IBGE. Dados amostragem por domicilio 2008. Contagem da população 2007. Censo 2000, censo 1982 [Internet]. [acesso em 10 maio 2008]. Disponível em: http://www.ibge.gov.br

4. Foss MP, Valle FAC, Speciali JG. Influência da escolaridade na avaliação neuropsicológica de idosos: aplicação e análise dos resultados da Escala de Mattis para Avaliação de Demência. Arq Neuropsiquiatr. 2005; 63(1): 119-26.

5. Smeltzer SC, Bare BG, Brunner \& Suddarth. Tratado de Enfermagem Médico-Cirúrgica. 10 ed. Rio de Janeiro: Guanabara Koogan; 2005

6. Siberman C, Souza C, Fabio W, Kipper L, Wu V, Diogo C, et al. Cognitive deficit and depressive symptoms in a community group of elderly people: a preliminary study. Rev Saúde Pública. 1995; 29(6): 444-50.

7. Carvalho Filho ET, Papaléo Neto M. Geriatria: fundamentos, clínica e terapêutico. 2. ed. São Paulo: Editora Atheneu; 2006.

8. Katon WJ. Clinical and health services relationships between major depression, depressive symptoms, and general medical illness. Biol Psychiatry. 2003; 54: 216-26.

9. Ferrari JF, Dalacorte RR. Use of Yesavage Geriatric Depression Scale to evaluate the prevalence of depression in inpatient elderly subjects. Sci Med. 2007; 17(1): 3-8.

10. American Psychiatric Association. DSM-IV-TR - Manual diagnóstico e estatístico de transtornos mentais. Trad. Claudia Dornelles. 4. ed. Porto Alegre: Artmed; 2002.
11. Scazufca M, Cerqueira ATAR, Menezes PR, Prince M, Vallada HP, Miyazaki, MCOS, et al. Investigações epidemiológicas sobre demências nos países em desenvolvimento. Rev Saúde Pública. 2002; 36(6): 773-8.

12. Machado JC, Ribeiro RCL, Leal PFG, Cotta RMM. Avaliação do declínio cognitivo e sua relação com as características socioeconômicas dos idosos em Viçosa-Mg. Rev Bras Epidemiol. 2007; 10(4): 592-605.

13. Herrera JRE, Caramelli P, Nitrini R. Estudo epidemiológico-populacional de demência na cidade de Catanduva, Estado de São Paulo, Brasil. Rev Psiquiatr. 1998; 25: 70-3.

14. Almeida OP, Almeida AS. Confiabilidade da versão Brasileira da escala de Depressão em Geriatria (GDS) versão reduzida. Arq Neuropsiquiatr. 1999; 57(2B): 421-6

15. Carvalho AM, Coutinho ESF. Demência como fator de risco para fraturas graves em idosos. Rev Saúde Pública. 2002; 36(4): 448-54.

16. Teng CT, Humes EC, Demetrio FN. Depression and medical comorbidity. Rev Psiquiatr Clin. 2005; 32(3): 149-59.

17. Scalco AZ, Scalco MZ, Azul JBS, et al. Hipertensão arterial sistêmica e depressão. Clinics. 2005; 60(3): 241-50

18. Musselmann DL, Betan E, Larsen H, Phillips LS. Relationship of Depression to Diabetes Types 1 and 2: Epidemiology, Biology and Treatment. Biol Psychiatry. 2003; 54: 317-29.

19. Bush DE, Ziegeltein RC, Tayback M, et al. Even Minimal Symptoms Depression Increase Mortality Risk after Acute Myocardial Infaction. Am J Cardiol. 2001; 88: 337-41.

20. Almeida-Pitito B, Almada Filho CM, Cendoroglo MS. Déficit cognitivo: mais uma complicação do diabetes melito. Arq Bras Endocrinol Metab. 2008; 52(7): 1073-83.

21. Okereke OI, Kang JH, Cook NR, Gaziano JM, Manson JE, Buring JE, et al. Type 2 Diabetes Mellitus and Cognitive Decline in Two Large Cohorts of Community-Dwelling Older Adults. J Am Geriatr Soc. 2008; 56: 1028-36.

22. Saxby BK, Harrington F, McKeith IG, Wesnes K, Ford GA. Effects of hypertension on attention memory, and executive function in older adults. Health Psychol. 2003; 22(6): 587-91.

23. Melo ROV, Martin JFV. Influência dos níveis pressóricos no desenvolvimento do déficit cognitivo. Rev Bras Hipertens. 2008; 15(1): 37-8.

24. Katz S, Ford AB, Moskowitz RW, Jackson BA, Jaffe MW. Studies of illness in the aged. The Index of ADL: a standardized measure of biological and psychosocial function. J Am Med Assoc. 1963; 165: 94-9.

25. Lawton MP, Brody EM. Assessment of older people: self-maintaining and instrumental activities of daily living. Gerontologist. 1969; 9: 179-86.

26. Yesavage JA, Brink TL, Rose TL, Lum O, Huang V, Adey M, et al. Development and validation of a geriatric depression screening scale: a preliminary report. J Psychiatr Res. 1982-1983; 17(1): 37-49.

27. Folstein MF, Folstein SE, Mchugh PR. Mini-Mental State: a practical method for grading the cognitive state of patients for clinician. J Psichiatry Res. 1975; 12:189-98.

28. Oliveira DAAP, Gomes L, Oliveira RF. Prevalência de depressão em idosos que freqüentam centros de convivência. Rev Saúde Pública. 2006; 40(4): 734-6.

29. Borges LJ, Benedetti TRB, Mazo GZ. Rastreamento cognitivo e sintomas depressivos em idosos. J Bras Psiquiatr. 2007; 56(4): 273-9.

30. Paradela EMP, Lourenço RA, Veras RP. Validação da escala de depressão geriátrica em um ambulatório geral. Rev Saúde Pública. 2005; 39(6): 918-23.

31. Engelhardt E, Laks J, Rozenthal M, Marinho VM. Idosos institucionalizados: rastreamento cognitivo. Rev Psiquiatr Clin. 1998; 25(2): 74-9.

32. Lourenco RA, Veras RP. Mini-exame do estado mental: características psicometricas em idosos ambulatoriais. Rev Saúde Pública. 2006; 40(4): 712-9.

33. Almeida Filho N, Lessa I, Magalhães L, Araujo MJ, Aquino E, James AS, et al. Social inequality and depressive disorders in Bahia, Brazil: interactions of gender, ethnicity and social class. Soc Sci Med. 2004; 59: 1339-53.

34. Pereira RJ, Cotta RMM, Franceschini SCC, Ribeiro RCL, Sampaio RF, Cecon PR. Contribuição dos domínios físico, social, psicológico e ambiental para a qualidade de vida global de idosos. Rev Psiquiatr Rio Gd Sul. 2006; 28(1): 27-38.

35. Colombini Neto M. Validade e coerência de instrumentos utilizados em avaliações clínicas de idosos. [dissertação]. Botucatu: Faculdade de Medicina da Universidade Estadual Paulista; 2008. 